\title{
On the Phase Response of the Error Diffusion Filter for Image Halftoning
}

\author{
Akhil Kumar and Anamitra Makur
}

\begin{abstract}
Conventional error diffusion halftoning uses a causal error filter. We propose the iterative error diffusion algorithm by extending the error diffusion to accommodate noncausal error filters. We realize the importance of the phase response of the error filter in the error diffusion halftoning method, and demonstrate it using examples. Iterative error diffusion is able to realize a zero phase error filter. We also trace a drawback of error diffusion to the shape of the error filter, and provide a remedy. The results obtained using zero phase error filter in the iterative error diffusion algorithm are, in our opinion, superior to the error diffusion halftones.
\end{abstract}

Index Terms - Error diffusion, image halftoning.

\section{INTRODUCTION}

$\mathbf{I}$ MAGE halftoning is the technique of rendering continuous tone images on display devices and printers that are bilevel, i.e., capable of reproducing only two levels, black and white. A halftone, though a binary image, possesses the appearance of continuous shades of gray owing to the way the black and white dots are distributed. Hence, the objective of halftoning is to create a halftone as much perceptually truthful to the continuous tone image as possible. While traditional halftones were analog halftones generated using various screening methods, today's digital image processing and multimedia utilities extensively use digital halftoning methods.

The invention of the error diffusion method [1] is regarded as a breakthrough in digital halftoning. For a halftone of a specified dot density, the error diffusion method produces better quality than methods invented before. Fig. 1 shows the block schematic of the error diffusion halftoning algorithm. Past errors $e(i, j)$ in converting a gray input pixel $y(i, j)$ to a binary value $g(i, j)$ are filtered and added (diffused) to the neighboring future pixels $f(i, j)$. It is a sequential single-pass algorithm. The error diffusion method may be interpreted using two-dimensional (2-D) signal processing, where the diffusion is understood to be a finite impulse response (FIR) filtering. The filter, referred to as the error filter, plays a crucial role in the performance of the algorithm.

There are a few classes of halftoning algorithms besides error diffusion. References [2] and [3] present a survey of these techniques. In [2], error diffusion is referred as the

Manuscript received July 14, 1996; revised December 8, 1998. The associate editor coordinating the review of this manuscript and approving it for publication was Dr. Ping Wah Wong.

A. Kumar is with IBM Global Services India Limited, Delhi, India.

A. Makur is with the Department of Electrical Communication Engineering, Indian Institute of Science, Bangalore 560012, India (e-mail: amakur@ece.iisc.ernet.in).

Publisher Item Identifier S 1057-7149(99)06831-1.

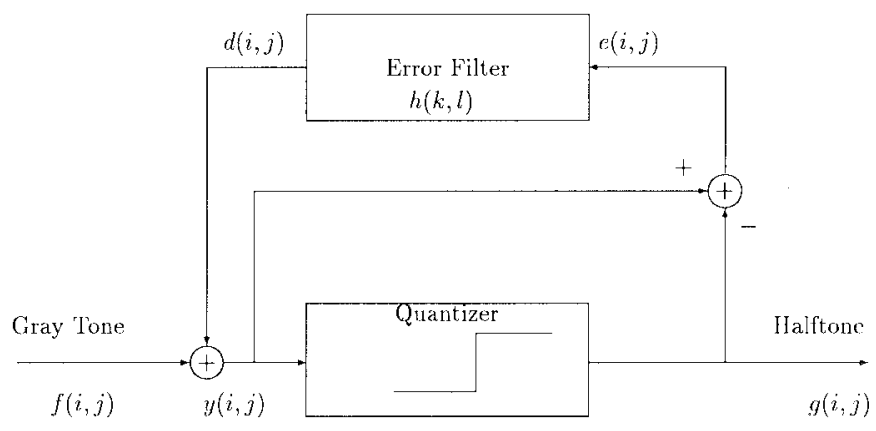

Fig. 1. Error diffusion method.

minimized average error technique. Till date, error diffusion remains an active area of research. Analysis of the error diffusion algorithm may be found in [4]-[6]. Ulichney showed that error diffusion generates blue noise which is desirable for halftoning [4]. Stucki used a larger error filter to disperse the objectionable patterns of error diffusion [7]. Fan proposed applying error diffusion on a halftone generated using the ordered dither [8]. In his method, error diffusion operates not on pixels but on ordered dither dots (except partial dots). The same author, in another paper, proposed reversing the scan order in error diffusion [9]. Both algorithms are examples of two-pass halftoning. Knox and Eschbach introduced threshold modulation by varying the threshold in error diffusion [10]. Such a scheme may be used for edge enhanced halftoning, image mixing, etc. Visual error diffusion suggested in [6] computes the error as the difference between the input and the visually blurred output halftone. Wong used least mean square algorithm to adapt the error filter based on the local intensity [11]. His approach improves the uniform regions while producing edges comparable to the conventional error diffusion.

\section{ITERATIVE ERROR DIFFUSION}

In this section, we propose an augmentation to the error diffusion procedure to accommodate noncausal error filters. A noncausal error filter may be realized if the future quantization error is available. We extend the error diffusion to an iterative (multipass) algorithm, so that the quantization error of one iteration (pass) may be collected and used during the next iteration as the quantization error of the future pixels. Though the quantization error from the last iteration will not be the exact quantization error of the future pixels in the current iteration, it indeed is a good estimate. 


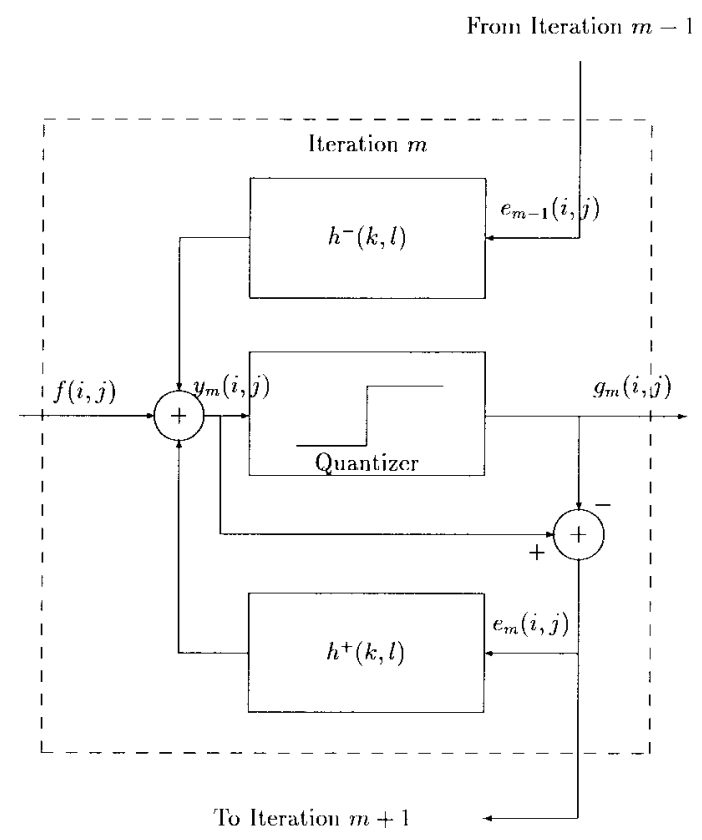

Fig. 2. One iteration of the iterative error diffusion method.

\section{A. Proposed Algorithm}

We refer to this extension of conventional error diffusion as the iterative error diffusion algorithm. An error image, containing some starting value of the quantization error for all pixels, is required. In each iteration the conventional error diffusion procedure is applied with the difference that a noncausal error filter is used instead of a causal error filter. The quantization error is updated on the error image on a pixel-by-pixel basis. As a result, the quantization errors for any iteration serve as the starting error values for the next iteration. The iterations are continued until a convergence criterion is satisfied. Fig. 2 depicts one iteration of the iterative error diffusion. The symbols $f, y, g$, and $e$ denote identical quantities to that of Fig. 1, and the subscripts indicate the iteration numbers. The error filter $h(k, l)$ has a full plane region of support, which is split into a strictly causal part $h^{+}(k, l)$ and the remaining (anticausal) part $h^{-}(k, l)$.

The iterative error diffusion algorithm needs a starting set of error values $e_{0}(i, j)$ (similar to the required starting halftone in the iterative Fourier transform algorithm [12]). Subsequently, the algorithm attempts to shape the feedback quantization error so that not only does it have the desired magnitude response, but also that it adds to the input signal in phase. This objective is achieved through a number of iterations or passes. We now show that the resulting halftoning noise does get shaped in a desirable way.

\section{B. Halftoning Noise in Error Diffusion}

It is possible to relate the halftoning noise to the error filter (similar prior work may be found in [6], [13], and [14]). Let $n(i, j)$ denote the halftoning noise, i.e., the difference between the actual image and the halftoned image

$$
n(i, j)=f(i, j)-g(i, j)
$$

with reference to Fig. 1. It follows that

$$
\begin{aligned}
n(i, j) & =e(i, j)-d(i, j) \\
& =e(i, j)-\sum_{(k, l) \in R} h(k, l) e(i-k, j-l)
\end{aligned}
$$

where $R$ is the region of support of the error filter. Taking the Fourier transform of both sides

$$
N\left(\omega_{x}, \omega_{y}\right)=E\left(\omega_{x}, \omega_{y}\right)\left(1-H\left(\omega_{x}, \omega_{y}\right)\right)
$$

where $\omega_{x}$ and $\omega_{y}$ represent the horizontal and vertical frequencies, respectively, and the capital letters represent the Fourier transforms of their lowercase counterparts (in [6, eq. (8)], also obtainable from [14, eq. (12a)]). Equation (3) relates the spectrums of the halftoning noise and the quantization error $e(i, j)$ to the error filter response. The halftoning noise spectrum, therefore, is shaped by $1-H\left(\omega_{x}, \omega_{y}\right)$. The quantization process may be modeled as a gain-plus-additive noise [15], where the noise is white and input-independent. Thus, the quantization error consists of a signal component and a white noise, and has a relatively flat power spectrum even for lowpass image input.

\section{Halftoning Noise in Iterative Error Diffusion}

The input to the quantizer during the $m$ th iteration is

$$
\begin{aligned}
y_{m}(i, j)= & f(i, j)+\sum_{(k, l) \in R_{f}} h^{+}(k, l) e_{m}(i-k, j-l) \\
& +\sum_{(k, l) \in R_{f}} h^{-}(k, l) e_{m-1}(i-k, j-l) .
\end{aligned}
$$

Here, $R_{f}$ is the full plane region of support. However, $h^{+}$ has zero coefficients outside the nonsymmetric causal half plane region of support excluding the origin, and $h^{-}$has zero coefficients outside the nonsymmetric anticausal half plane region of support including the origin. Therefore, $h^{+}$works only on $e_{m}(i, j)$, the error produced in the current iteration, while $h^{-}$works only on $e_{m-1}(i, j)$, the error of last iteration. Since $e_{m}(i, j)=y_{m}(i, j)-g_{m}(i, j)$, it follows from (4) that

$$
\begin{aligned}
E_{m}\left(\omega_{x}, \omega_{y}\right)= & F\left(\omega_{x}, \omega_{y}\right)-G_{m}\left(\omega_{x}, \omega_{y}\right) \\
& +H^{+}\left(\omega_{x}, \omega_{y}\right) E_{m}\left(\omega_{x}, \omega_{y}\right) \\
& +H^{-}\left(\omega_{x}, \omega_{y}\right) E_{m-1}\left(\omega_{x}, \omega_{y}\right)
\end{aligned}
$$

where the capital letters denote the Fourier transforms of there lowercase counterparts. If the spectrum of the halftoning noise during the $m$ th iteration is denoted by $N_{m}\left(\omega_{x}, \omega_{y}\right)$, then

$$
\begin{aligned}
N_{m}\left(\omega_{x}, \omega_{y}\right)= & \left(1-H^{+}\left(\omega_{x}, \omega_{y}\right)\right) E_{m}\left(\omega_{x}, \omega_{y}\right) \\
& -H^{-}\left(\omega_{x}, \omega_{y}\right) E_{m-1}\left(\omega_{x}, \omega_{y}\right) .
\end{aligned}
$$

The feedback error spectrum gets shaped as the iterations proceed. If this shape converges after $m$ iterations, then

$$
E_{m-1}\left(\omega_{x}, \omega_{y}\right) \approx E_{m}\left(\omega_{x}, \omega_{y}\right) \approx E\left(\omega_{x}, \omega_{y}\right) .
$$

Therefore, after convergence

$$
N_{m}\left(\omega_{x}, \omega_{y}\right)=E\left(\omega_{x}, \omega_{y}\right)\left(1-H\left(\omega_{x}, \omega_{y}\right)\right) .
$$

This relation is identical to that in (3). 
In practice, convergence is achieved when a resulting halftone has no perceptual difference from the previous halftone. This happens when the changes between two halftones $g_{m}(i, j)$ and $g_{m-1}(i, j)$ take place only in the high frequency regions. Let the difference between two consecutive halftones be

$$
\tilde{n}_{m}(i, j)=g_{m-1}(i, j)-g_{m}(i, j) .
$$

Instead of assuming the convergence of the feedback error spectrum in (7), a good approximation after a few iterations is

$$
\begin{aligned}
& E_{m}\left(\omega_{x}, \omega_{y}\right)-E_{m-1}\left(\omega_{x}, \omega_{y}\right) \\
& \quad \approx E_{m-1}\left(\omega_{x}, \omega_{y}\right)-E_{m-2}\left(\omega_{x}, \omega_{y}\right) \\
& \quad \approx \tilde{E}\left(\omega_{x}, \omega_{y}\right)
\end{aligned}
$$

which means that the feedback error spectrum changes by some given amount during each iteration after, say, $m$ iterations. It follows from (6) that

$$
\tilde{N}_{m}\left(\omega_{x}, \omega_{y}\right)=\tilde{E}\left(\omega_{x}, \omega_{y}\right)\left(1-H\left(\omega_{x}, \omega_{y}\right)\right) .
$$

Since $\left(1-H\left(\omega_{x}, \omega_{y}\right)\right)$ is a highpass filter, the last relation shows that after a few initial iterations, the changes from one halftone to another are limited to the high frequency region. This confirms the observed fact that the output halftones from different iterations look similar, because the high frequency changes are not noticeable to the human eye in the case of halftones. This fact will be illustrated later by example.

\section{Initialization and Convergence}

The iterative error diffusion algorithm is initialized using a starting set of quantization error values. Any starting value such as all zeros may be used. However, for faster convergence, it is desirable that the values are limited to $(-1 / 2,1 / 2)$ with a zero mean (where 1 is the maximum intensity value that any pixel in the input can have), and that the spectrum contains high frequency components. Some possible choices satisfying these conditions are highpass noise and white noise. An even faster convergence may be achieved when the starting values are the quantization errors from a good halftone.

It has already been pointed out that a suitable convergence criterion for the iterative error diffusion algorithm should be based on the change in the output halftone. A numerical measure for this change is the energy of $\tilde{n}(i, j)$. Since $\tilde{n}(i, j)$ takes a value of $-1,0$, or 1 , its energy is proportional to the fraction of pixels that change their values between two iterations. It has been observed that this fraction depends on the error filter but is rather insensitive to the input image. A typical convergence threshold for this fraction is 0.06 . Typically after ten iterations, the fraction of changed pixels falls below this threshold, and no significant perceptual improvement or decrease in the fraction value is observed subsequently.

\section{Zero Phase ERror FiLter}

Since iterative error diffusion allows use of noncausal error filters, we propose using a zero phase error filter to exploit this added flexibility. In the next section we illustrate the importance of the phase response of the error filter.

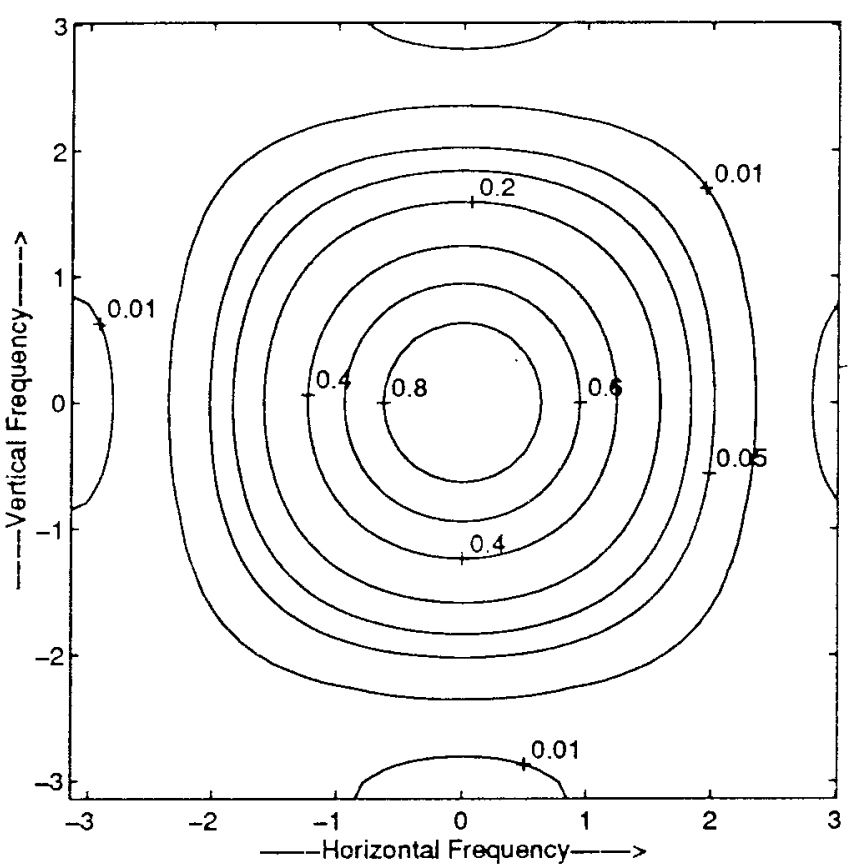

(a)

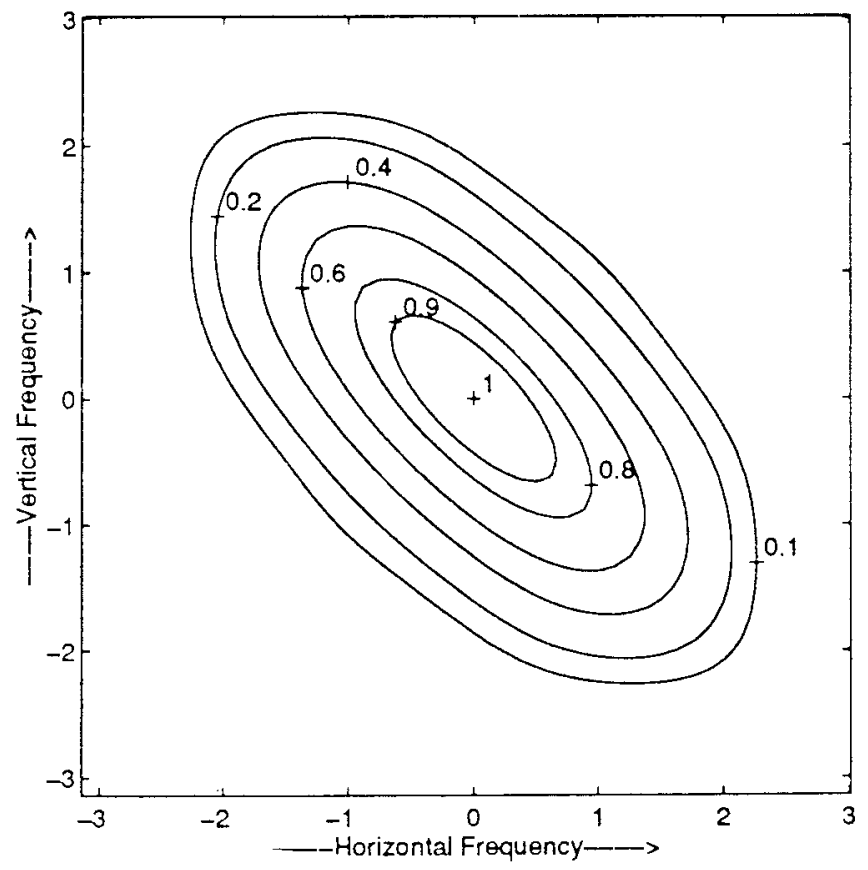

(b)

Fig. 3. Magnitude response of the error filters of example 1: (a) $h_{1}$ and $h_{2}$ and (b) $h_{3}$

\section{A. Magnitude Versus Phase}

The error filter is situated at the feedback path. The quantization error, after passing through this filter, is added to the input image as a corrective measure. The response of the error filter, therefore, determines which part of the input image spectrum is retained by the halftoning method, and which part is not. We shall illustrate this fact later with respect to both the magnitude and the phase spectrum. If the magnitude response of the error filter is lowpass with unity gain as zero frequency, it ensures 
that the low frequency spectrum of the halftone is similar to that of the input image. If the error filter is zero phase, it ensures that the error that has been fed back is added in phase with the input image. While a lowpass response is desirable in order to simulate the human visual system, a zero phase response is desirable in order to retain the sharp edges. The phase response of the error filter is more important than its magnitude response for halftoning. We demonstrate this fact using the following example.

Example 1: Consider the following three error filters in (12)-(14), shown at the bottom of the page. $h_{1}$ is a separable 2D lowpass zero phase filter and $h_{2}$ is a separable 2-D lowpass filter having identical magnitude response as $h_{1}$. However, the phase response of $h_{2}$ differs from that of $h_{1}$ since $h_{2}$ is not a zero phase filter. $h_{3}$ is a nonseparable 2-D lowpass zero phase filter having identical phase response as $h_{1}$. The magnitude response of $h_{3}$ differs from that of $h_{1}$. Fig. 3 shows the magnitude responses of $h_{1}$ (or $h_{2}$ ) and $h_{3}$. Fig. 4 shows the halftones obtained using filters $h_{1}, h_{2}$, and $h_{3}$, on the popular image Lenna using the iterative error diffusion. While the output halftones of Fig. 4(a) and (c) are comparable, the halftone of Fig. 4(b) is clearly unacceptable. The radially averaged absolute phase error between the original image and the halftone is plotted in Fig. 5 for all three cases. The phase error is unwrapped to within an interval $-\pi$ to $\pi$ before taking the absolute value, and the 2-D spectrum has been radially averaged. Observe that the zero phase filters $h_{1}$ and $h_{3}$ produce considerably less mean absolute phase error at the low frequencies than the nonzero phase filter $h_{2}$. This figure illustrates that the phase response of the error filter determines which part of the input image phase spectrum is retained by the halftoning method.

The above example shows that, at least in this case, the phase response is more important than the magnitude response of the error filter. It is only desired that the magnitude response should be a lowpass, preferably with its pass band not less than the passband of the human visual system. A nonzero phase error filter, however, introduces a phase distortion to the error feedback so that the input and the feedback error do not add in correct phase. The result of this out-of-phase addition is that it tends to place the halftone dots at wrong locations. It results in the loss of fine details in the output halftone and creates edges which are rough. When the human eye perceives these rough edges, they look blurred because of the extended width of the edges. Various researchers have referred to this blur. Ulichney calls it the unsharpening degradation, or the transient behavior near abrupt edges, and includes it as one of the three defects of error diffusion [4]. This phenomenon is also identified as a drawback of error diffusion and referred as diffusion across edges [6] or bad rendition of edges [16].

Edge blurring should be distinguished from the edge enhancement in error diffusion pointed out in [17]-[20]. While edge blurring refers to the deterioration of edge slope, edge enhancement refers to the enhancement of edge contrast. Eschbach and Knox experimentally shows the occurrence of edge blurring and edge enhancement in error diffusion in [17, Fig. 6(a)], where both slope degradation and contrast enhancement are observed.

Even though the fact that zero (or linear) phase is essential in image processing application to preserve edges is well known, to our surprise we did not find any explicit mention of zero phase error filter in halftoning literature. The reason perhaps is that such a filter is not implementable in a single-pass algorithm using a sequential scanning. Several researchers, however, used noncausal filters and phase constraints in halftoning. Kollias and Anastassiou realized that "bad rendition of fine edges" in the error diffusion is "related to the unidirectional processing," and suggested a "symmetric" noncausal error filter as a remedy [16]. They subsequently used neural network to bypass the single-pass limitation. Note that their proposed quadrant-symmetric error filter is more restrictive than a zero phase error filter. In order to achieve a "more symmetric error distribution" than is possible using a sequential scan, Fan proposed a two-pass error diffusion where the second pass uses a reverse scanning order [9]. The author states that such an algorithm realizes a symmetric magnitude response of $\left(1-H\left(\omega_{x}, \omega_{y}\right)\right)$. Katsavounidis and Kuo used a noncausal error filter, but their scheme realizes an order statistics filtering rather than a linear filtering [21]. The scanning order used by them is based on the intensity, and due to multiscale processing the required complexity is $\log N$ times more than a single-pass processing (where $N \times N$ is the size of the image). Bryngdahl and group have found in [22] and [23] that introducing some phase constraints in the iterative Fourier transform algorithm for halftoning [12] leads to some improvements.

\section{B. Drawbacks of the Error Diffusion}

The error diffusion method is known to have two drawbacks, edge blurring (degradation of the edge slope), and worm artifacts in uniform highlight or shadow regions. Worms are the periodic artifacts in low-modulation image regions [6] that are also referred as the avalanche patterns [3]. We are not aware of any attempt to pinpoint the reason for these drawbacks, even though investigators have looked into modifications to

$$
\begin{aligned}
& h_{1}=\left[\begin{array}{lllll}
0.0750 & 0.2462 & 0.3576 & 0.2462 & 0.0750
\end{array}\right]^{t} \cdot\left[\begin{array}{llllll}
0.0750 & 0.2462 & 0.3576 & 0.2462 & 0.0750
\end{array}\right] \\
& h_{2}=\left[\begin{array}{lllllllll}
0.1171 & 0.3003 & 0.3424 & 0.1922 & 0.0480
\end{array}\right]^{t} \cdot\left[\begin{array}{lllll}
0.1171 & 0.3003 & 0.3424 & 0.1922 & 0.0480
\end{array}\right] \\
& h_{3}=\left[\begin{array}{rrrrr}
0.0375 & 0.0356 & 0.0040 & -0.0136 & 0.0071 \\
0.0356 & 0.1045 & 0.0980 & 0.0249 & -0.0136 \\
0.0040 & 0.0980 & 0.1560 & 0.0980 & 0.0040 \\
-0.0136 & 0.0249 & 0.0980 & 0.1045 & 0.0356 \\
0.0071 & -0.0136 & 0.0040 & 0.0356 & 0.0375
\end{array}\right]
\end{aligned}
$$




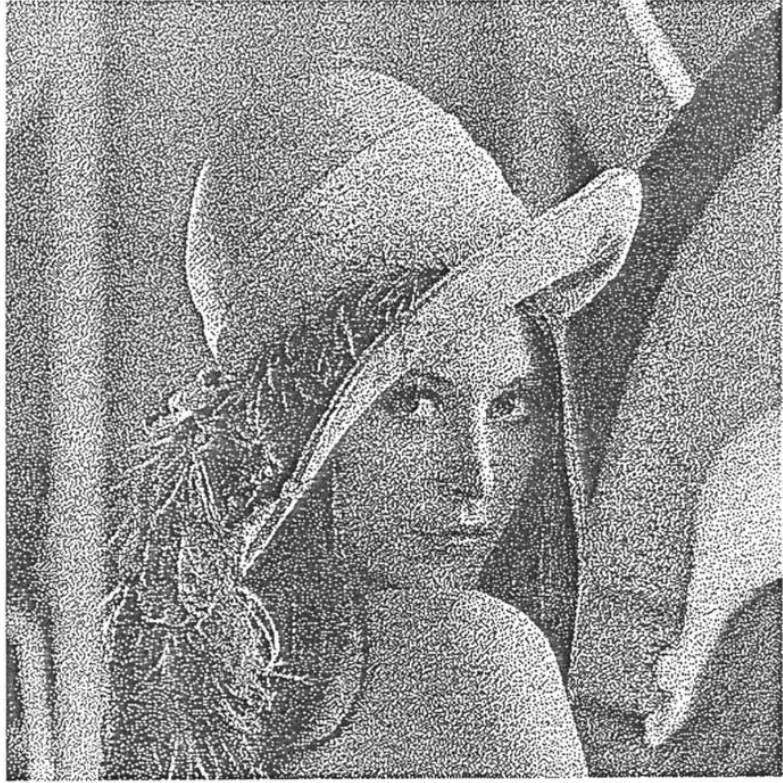

(a)

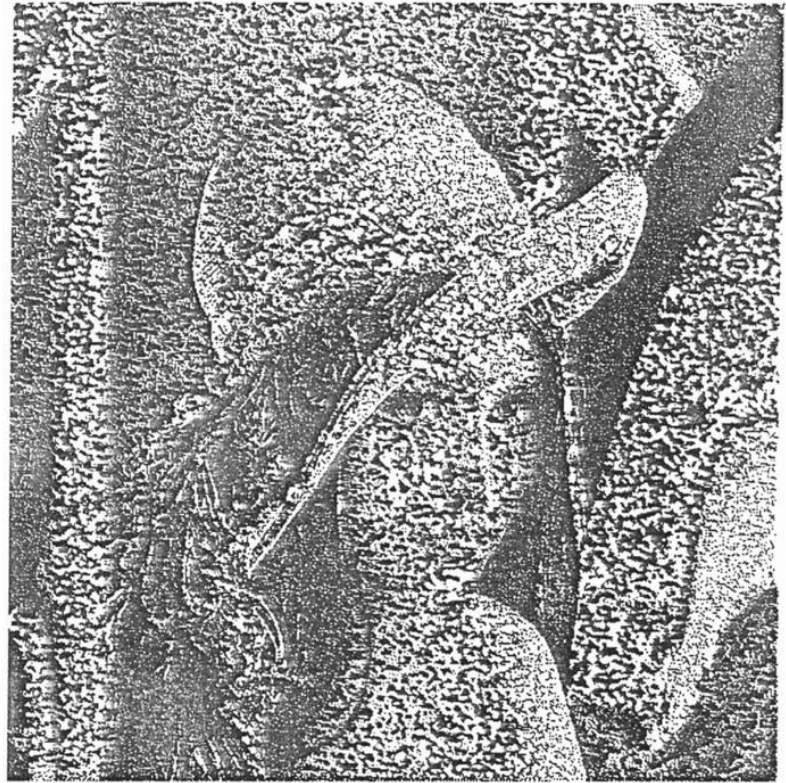

(b)

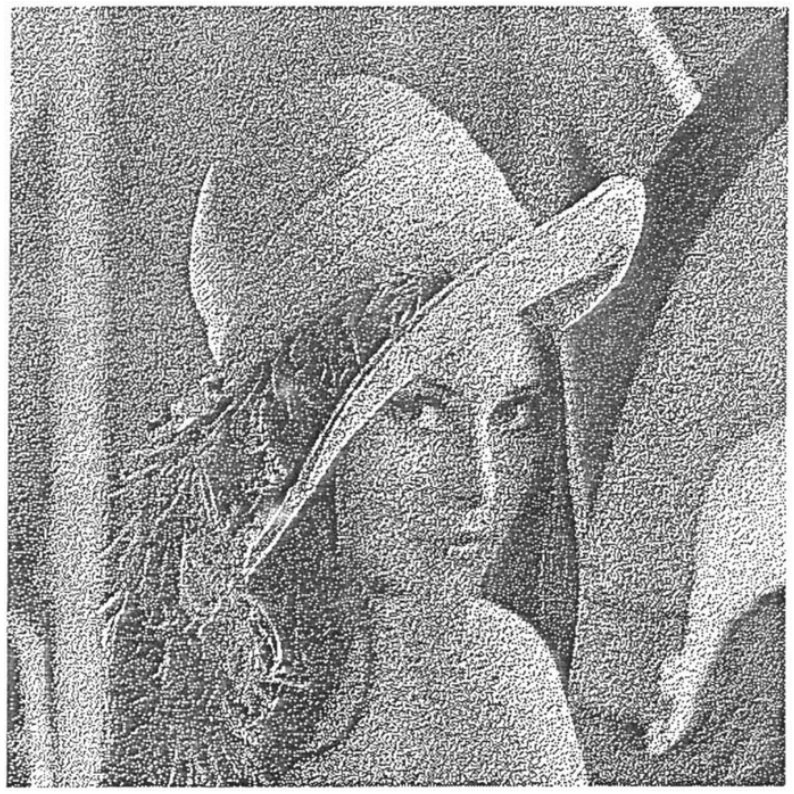

(c)

Fig. 4. Halftone using the error filters of example 1: (a) $h_{1}$, (b) $h_{2}$, and (c) $h_{3}$.

overcome these shortfalls. For example, Ulichney mentions use of sharpening to improve, or at least defeat, unsharpening degradation [4]; in [6] a brief survey of works toward reducing worms such as that of Stucki [7] is provided. We attribute both these drawbacks to the response of the error filter. Specifically, as argued in the previous section, nonzero phase response is responsible for the edge blurring. We now examine the worm artifacts.

Refer to (3). The magnitude response of $H\left(\omega_{x}, \omega_{y}\right)$ is typically a lowpass filter modeling the human visual response. Since a conventional error filter is strictly causal, $H\left(\omega_{x}, \omega_{y}\right)$ is not zero (or linear) phase. Consequently, $1-H\left(\omega_{x}, \omega_{y}\right)$ does not have a shape which is exactly the complement of $H\left(\omega_{x}, \omega_{y}\right)$, but it is a nonzero phase high pass filter. In this work we have used a $3 \times 5$ error filter having the following impulse response (diffusion weights)

$\begin{array}{ccccc}0 & 0 & 0 & 0.15 & 0.10 \\ 0.06 & 0.10 & 0.15 & 0.10 & 0.06 \\ 0.03 & 0.06 & 0.10 & 0.06 & 0.03\end{array}$

where 0 denotes the $(0,0)$ th coefficient. The magnitude response of this filter is shown in Fig. 6. The magnitude response of the corresponding $1-H\left(\omega_{x}, \omega_{y}\right)$ is shown in Fig. 7. The magnitude of the power spectrum of the halftoning noise for Lenna using the conventional error diffusion is shown in Fig. 8. Contour plots are used for this and latter power spectrums, since they nicely discriminate between the lowenergy region (shown as the blank space) and high-energy 


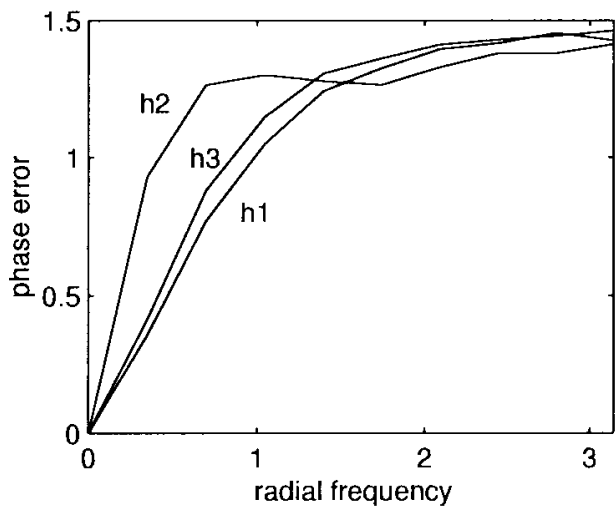

Fig. 5. Mean absolute phase error using the error filters of example 1.

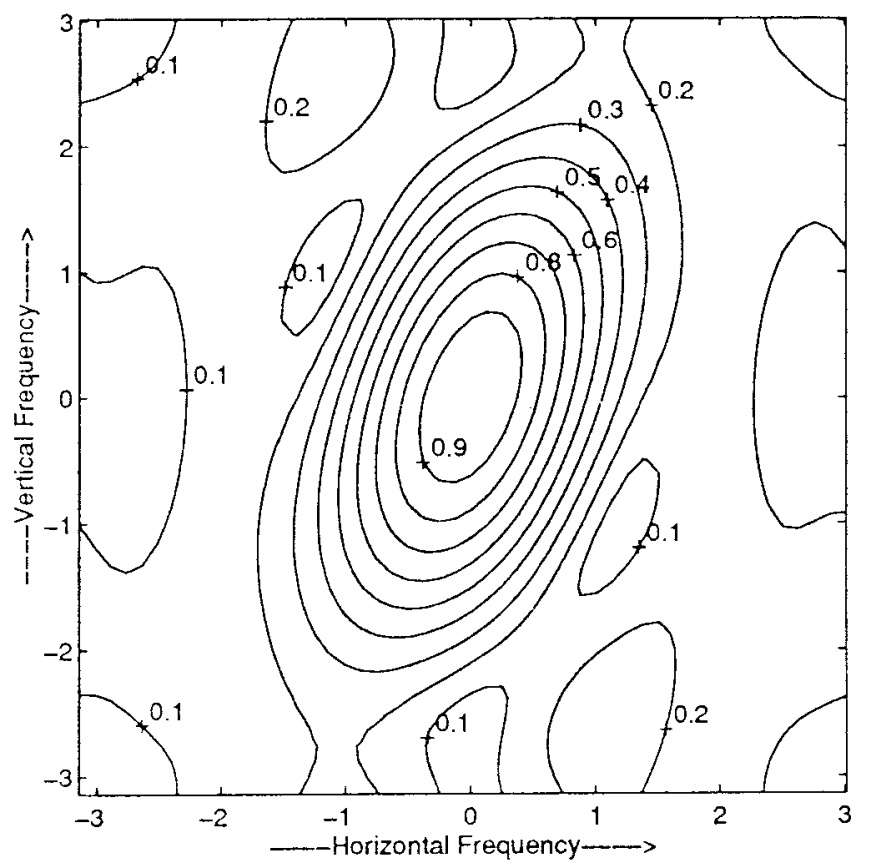

Fig. 6. Magnitude response of the error filter of (15).

noiselike region (with dense variations, shown as various contours). The shape of the halftoning noise is indeed in agreement with the magnitude response of $1-H\left(\omega_{x}, \omega_{y}\right)$. In Fig. 7, the magnitude response profile along a few radial directions, such as $\pi / 2$ and 0 , has the following shape. It increases from zero at low frequency, to a high value at midfrequency, and then falls to a lower value at high frequency. This shape is in agreement with the error spectrum shown in [6, Fig. 6] and [4, Fig. 13]. This shape gives rise to band pass halftoning noise in these directions (see Fig. 8). Such a halftone is expected to have alternate stripes of these orientations. In uniform regions of the image, such perceptible stripes are the worm artifacts. To illustrate our point, we once again present an example.

Example 2: Consider the following two error filters:

$$
h_{4}=\left[\begin{array}{ccccc}
0 & 0 & 0 & 0.083 & 0.083 \\
0.083 & 0.083 & 0.083 & 0.083 & 0.083 \\
0.083 & 0.083 & 0.083 & 0.083 & 0.083
\end{array}\right]
$$

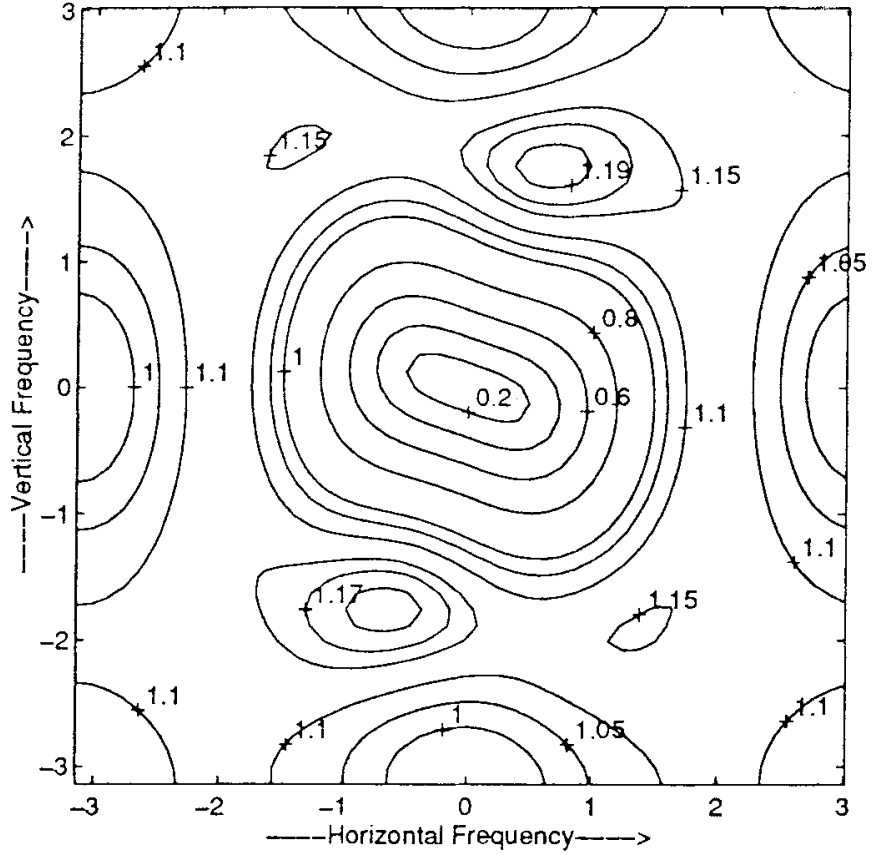

Fig. 7. Magnitude response of $1-H\left(\omega_{x}, \omega_{y}\right)$ of (15).

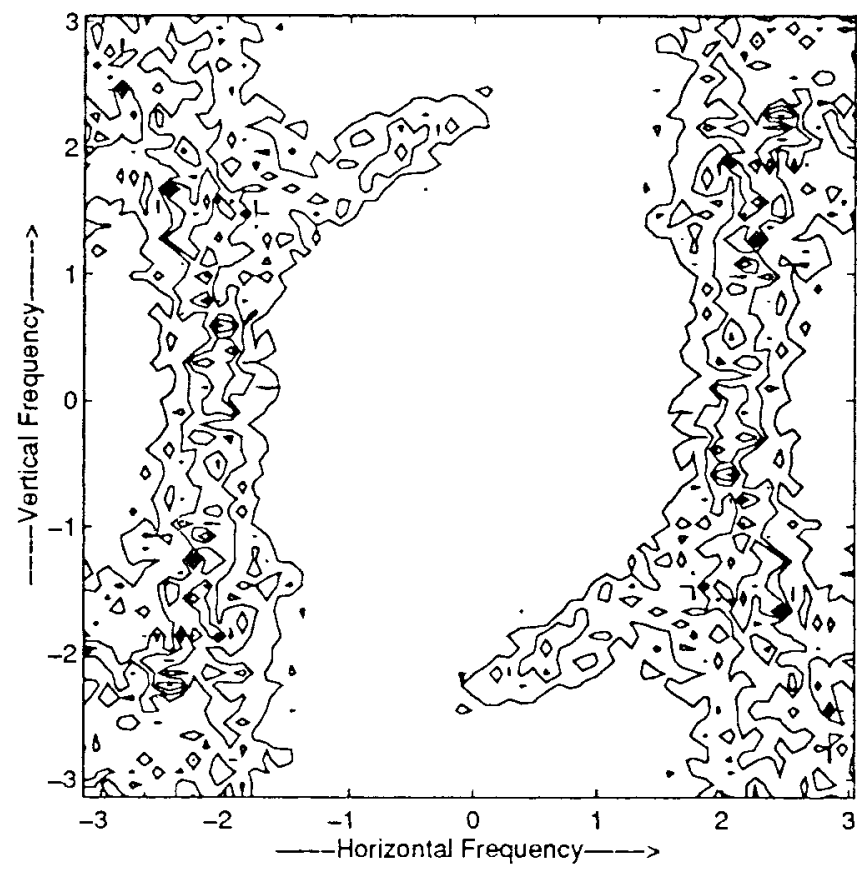

Fig. 8. Power spectrum of the halftoning noise for (15).

which results in $1-H\left(\omega_{x}, \omega_{y}\right)$ having prominent bandpass shape at horizontal and vertical frequencies, and

$$
h_{5}=\left[\begin{array}{ccccr}
0 & 0 & 0 & 0.2700 & -0.0621 \\
-0.0947 & 0.0518 & 0.2974 & 0.3026 & 0.0603 \\
0.0076 & -0.0504 & 0.0096 & 0.1046 & 0.1033
\end{array}\right]
$$

which produces $1-H\left(\omega_{x}, \omega_{y}\right)$ having no bandpass nature. Applying these error filters on the same image results in the halftones, which are shown in Fig. 9. The corresponding 


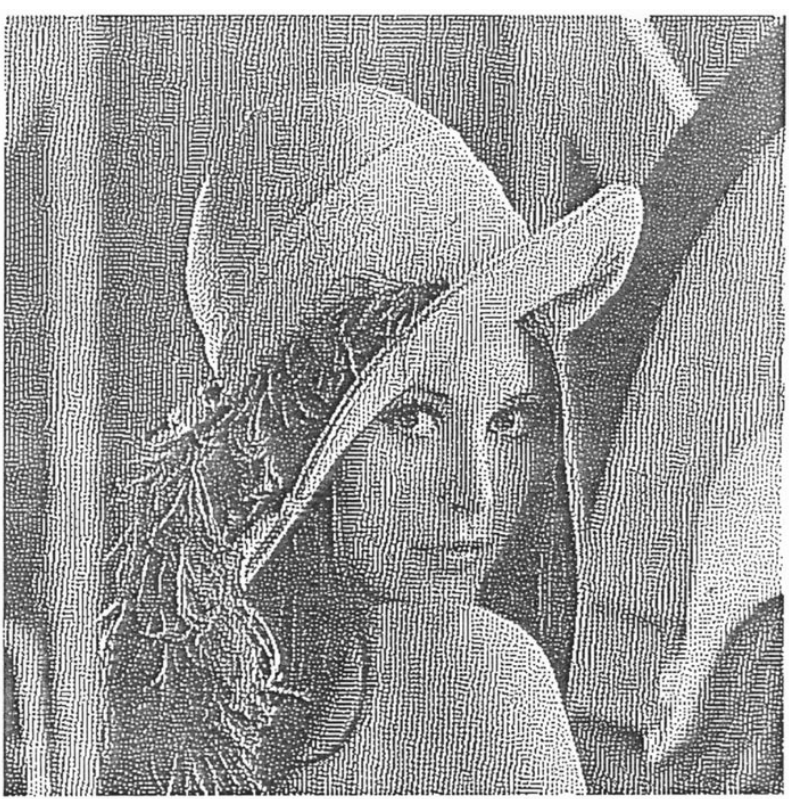

(a)

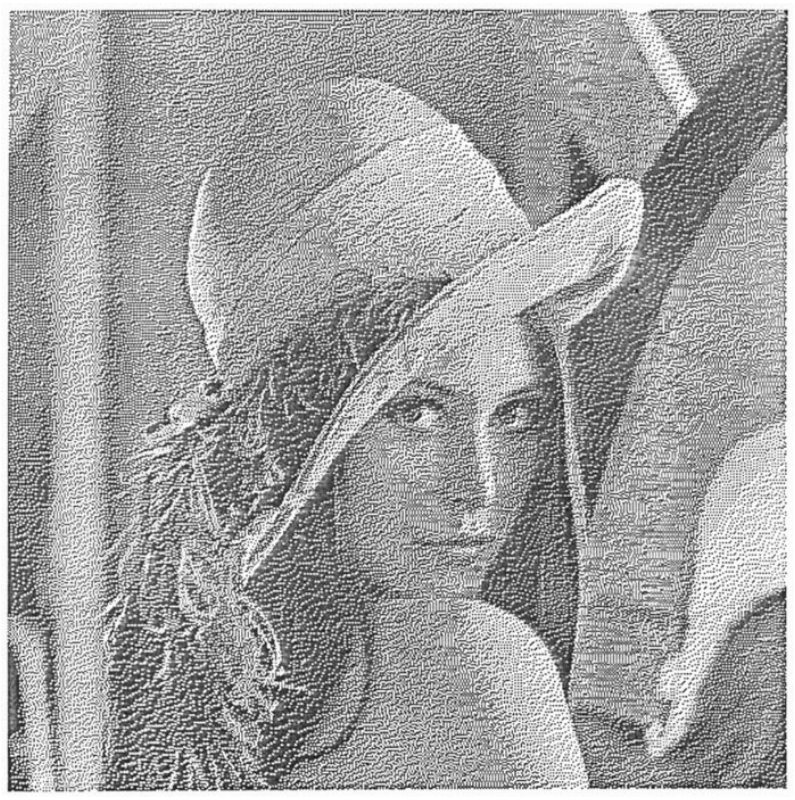

(b)

Fig. 9. Halftone using the error filters of example 2: (a) $h_{4}$ and (b) $h_{5}$.

halftoning noise power spectrums are shown in Fig. 10. While $h_{4}$ produces very prominent worms in horizontal and vertical directions, $h_{5}$ produces almost no worms.

We conclude that, since a bandpass shape of $1-H\left(\omega_{x}, \omega_{y}\right)$ in error diffusion results in worms, such a shape should be avoided.

\section{Choosing the Error Filter for Iterative Error Diffusion}

In conventional error diffusion, the error is fully diffused to future pixels. Let this be referred to as diffusion in space. Such a diffusion has the ability to retain the average intensity over space. This property is termed differently by researchers as no error leakage [21] and mean preservable. If the error is

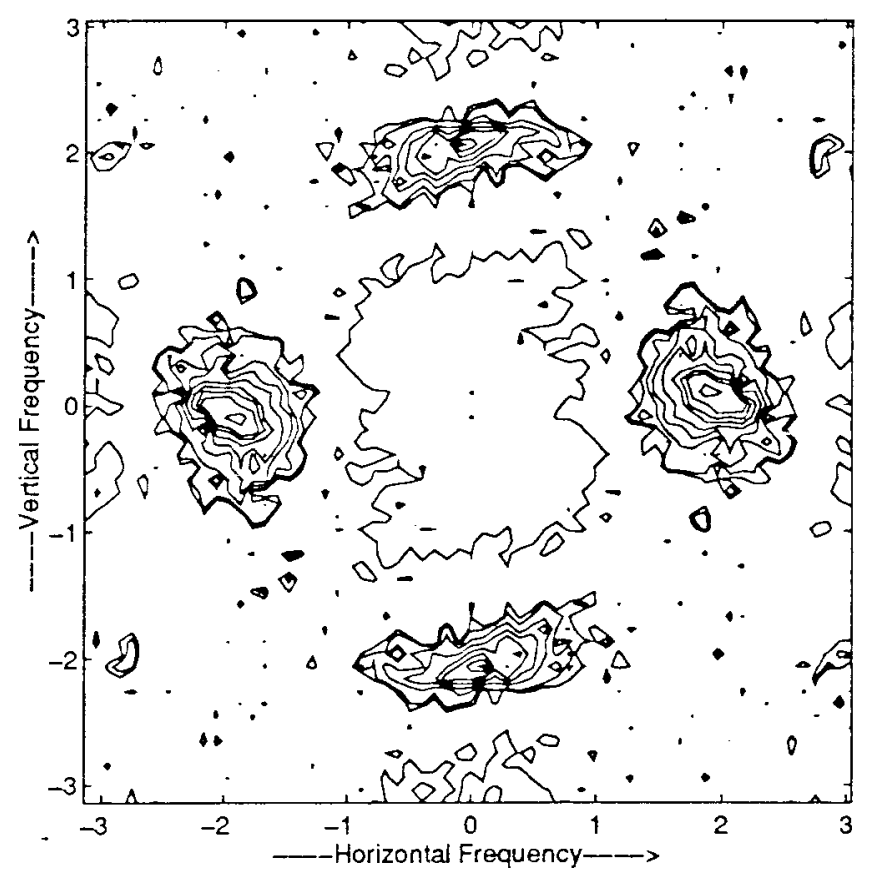

(a)

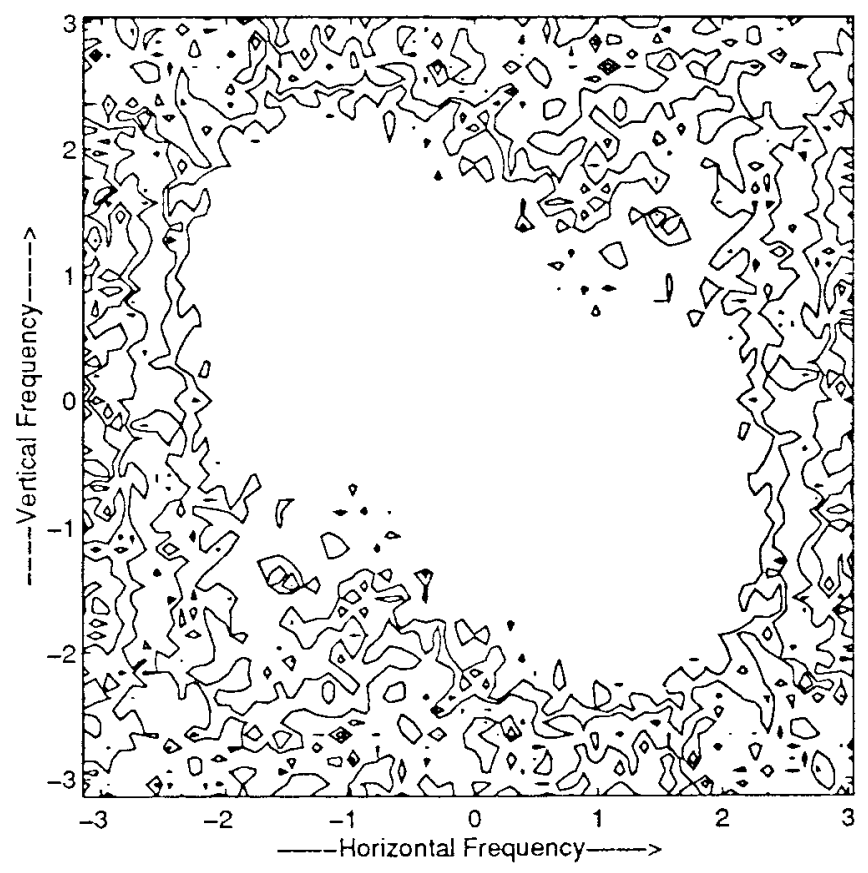

(b)

Fig. 10. Power spectrum of the halftoning noise for example 2: (a) $h_{4}$ and (b) $h_{5}$.

not fully diffused, the remaining part of the error is not compensated by neighboring pixels; the error keeps accumulating at every pixel.

In iterative error diffusion, diffusion occurs also in time (iteration). An error filter having $h^{-}(0,0)=1$ and remaining coefficients 0 will diffuse the error fully in time. In such a case, a pixel intensity is indeed preserved over time (that is, over the average of various output halftones produced in consecutive iterations). For example, a gray value of 0.9 would produce 


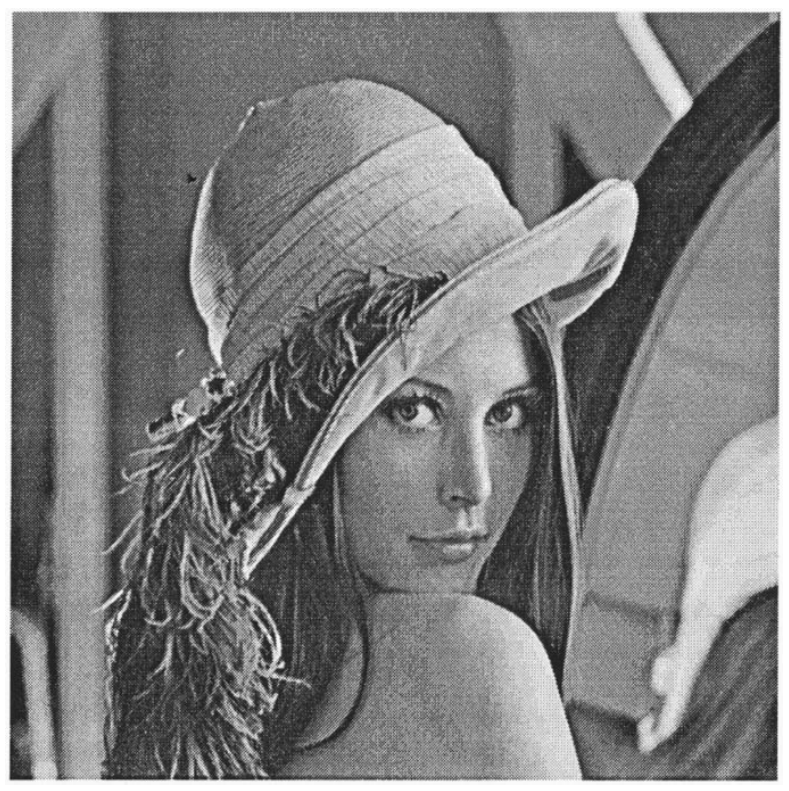

Fig. 11. Input image of Lenna.

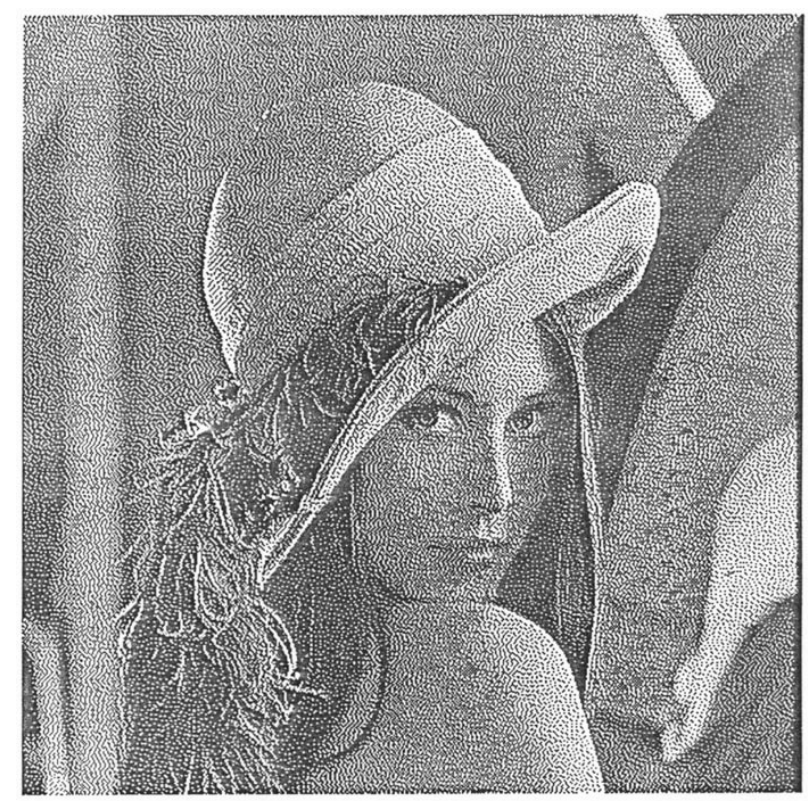

Fig. 12. Halftone using the error filter of (15).

nine white dots and one black dot in a period of ten iterations. However, averaging in space is acceptable since the human eye does the same. Averaging in time does not serve the purpose since a viewer looks only at any individual iteration output. It may be concluded that diffusion in space is preferred for halftoning than diffusion in time. The coefficient at the origin, $h^{-}(0,0)$, is capable of diffusion in time only, which is not desirable. Such a diffusion can be avoided by making this coefficient zero. Having a nonzero coefficient at the origin leads to a halftoning noise with considerable low frequency energy, which is easily perceived by the human eye.

It may be noted here that due to the presence of diffusion in time, absolute convergence is not expected in iterative error diffusion, merely the average halftone over space-time is converged.

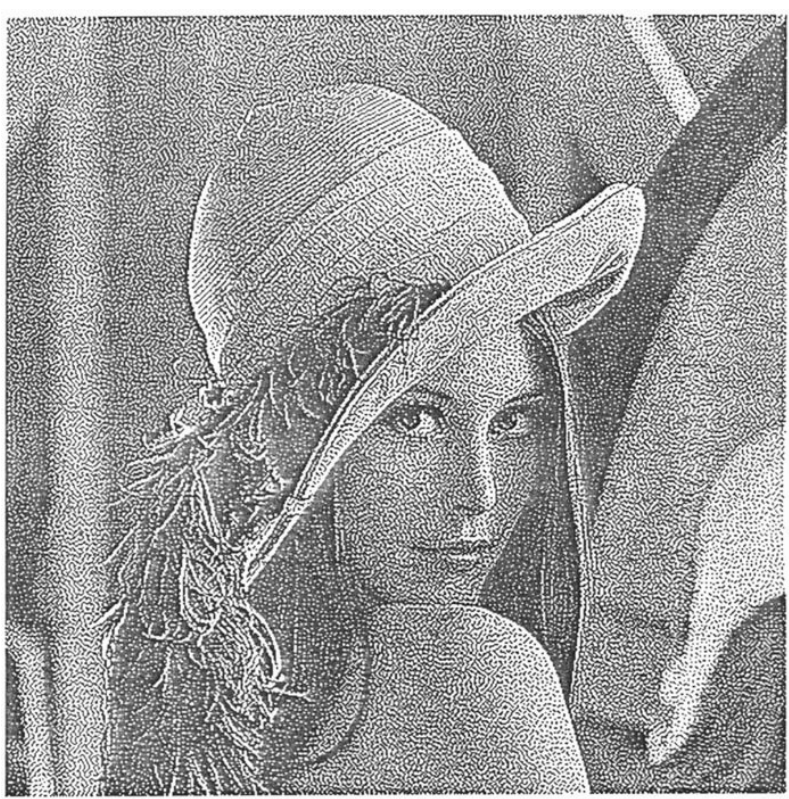

Fig. 13. Halftone using the error filter of (18) after 10 iterations, starting with the error diffusion error.

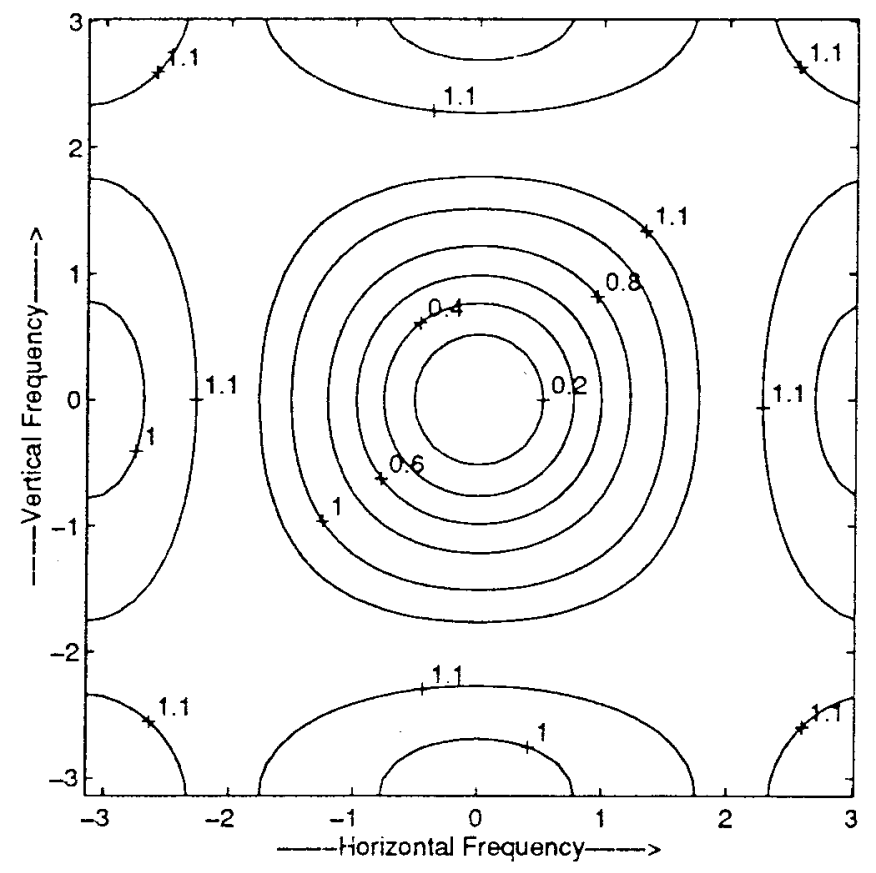

Fig. 14. Magnitude response of $1-H\left(\omega_{x}, \omega_{y}\right)$ of (18).

The error filter we choose is a zero phase filter. Because of the opposite quadrant symmetry property of 2-D zero phase filters, two parts of the filter $h^{+}(k, l)$ and $h^{-}(k, l)$ will have same coefficients except for the coefficient at the origin which will be present only in the second part (which we take as zero). The filter should be lowpass with unity gain at zero frequency. Let the desired shape of the halftoning noise be given. Equating this shape to $1-H\left(\omega_{x}, \omega_{y}\right)$, the required shape of the magnitude response of $H\left(\omega_{x}, \omega_{y}\right)$ may be obtained easily since now it is zero phase. A procedure to design the error filter having a shape conforming to the image spectrum is suggested in [24]. 


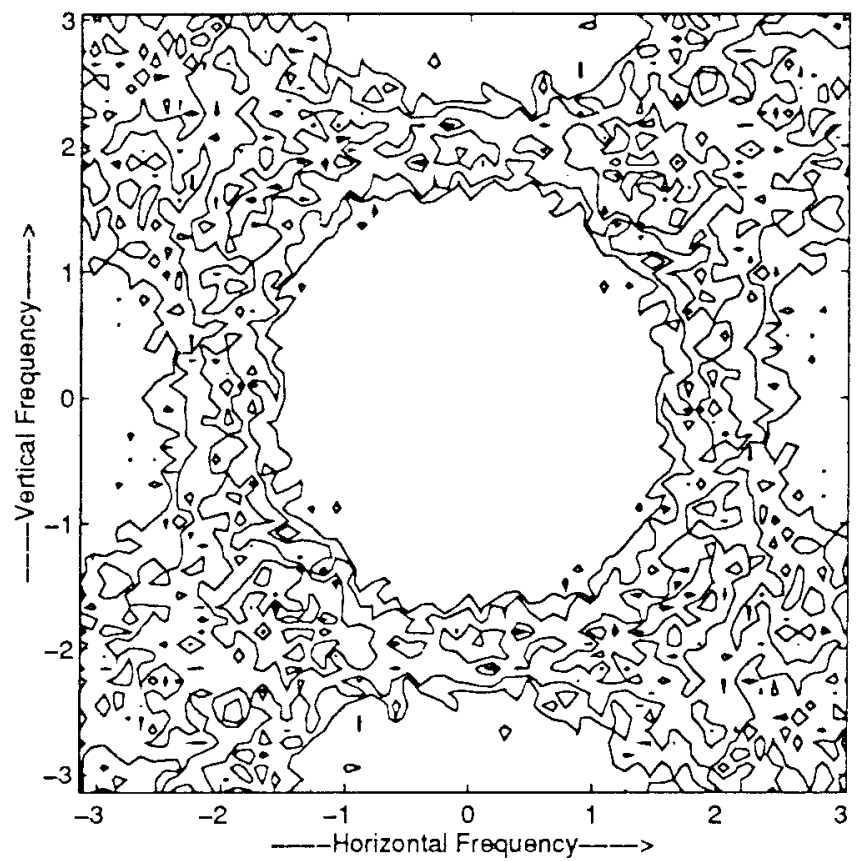

Fig. 15. Power spectrum of the halftoning noise for Fig. 13.

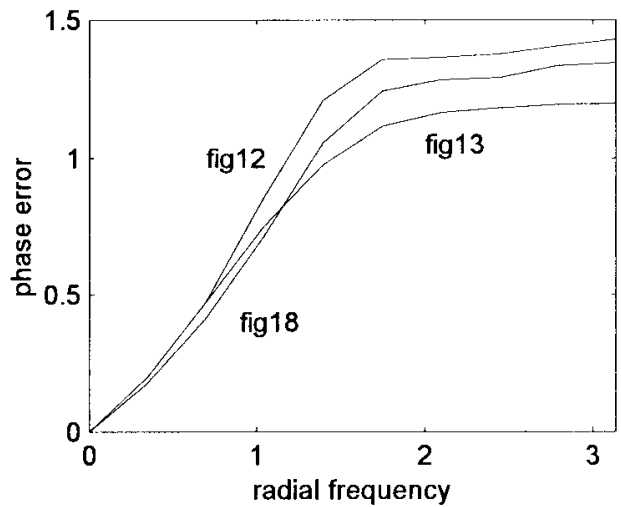

Fig. 16. Mean absolute phase error for Figs. 12, 13, and 18.

\section{RESUlTS AND CONCLUSION}

In order to compare our simulations with the conventional error diffusion, we used a zero phase version of the error filter of (15) for the proposed iterative error diffusion algorithm. Its impulse response is

$\left[\begin{array}{ccccc}0.015 & 0.030 & 0.050 & 0.030 & 0.015 \\ 0.030 & 0.050 & 0.075 & 0.050 & 0.030 \\ 0.050 & 0.075 & 0 & 0.075 & 0.050 \\ 0.030 & 0.050 & 0.075 & 0.050 & 0.030 \\ 0.015 & 0.030 & 0.050 & 0.030 & 0.015\end{array}\right]$.

Fig. 11 shows the input image Lenna, retaining almost all the features of the gray tone. In contrast, all halftones have a dot density of one dot per pixel. Fig. 12 shows the halftone obtained from the conventional error diffusion method using the error filter of (15). Fig. 13 shows the halftone obtained from the proposed iterative error diffusion method using the zero phase error filter, after ten iterations. The starting error values are the errors obtained from the conventional error

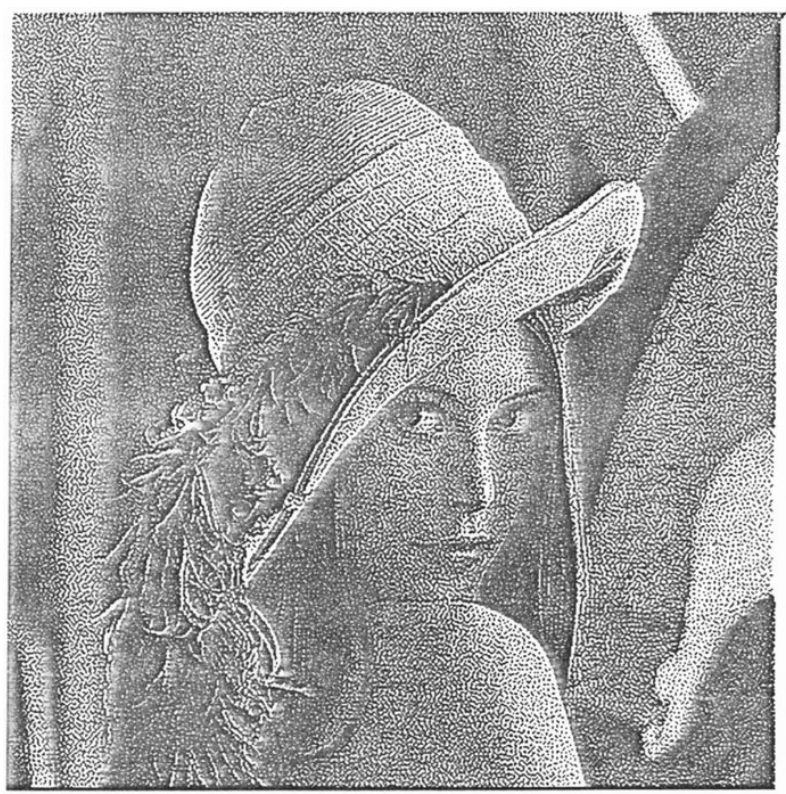

Fig. 17. Halftone using the error filter of (18) after ten iterations, starting with random error.

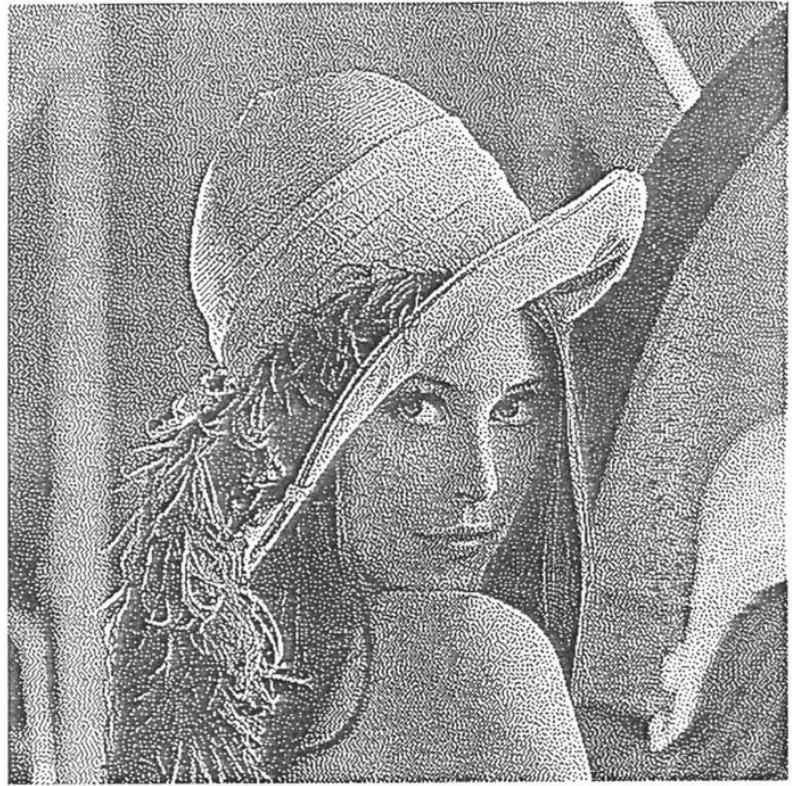

Fig. 18. Halftone using the error filter of (18) after one iteration, starting with the error diffusion error.

diffusion method. The effect of using the zero phase filter can be observed in all the regions which have fine details. One example is the lines in the upper parts of the hat which were all missing in Fig. 12. Similarly in the lower parts of the hat which has a scarf tied around the hat, the folds of the scarf are very prominent in Fig. 13. There is a line going along the rim of the hat which is not visible in Fig. 12 but it can be clearly seen in Fig. 13. Fig. 13 reproduces even very thin edges compared to the other halftone. All these examples establish that the zero phase error filter is much more truthful in reproducing the fine details of the actual image. Due to the zero phase response, this effect is symmetric, unlike the 


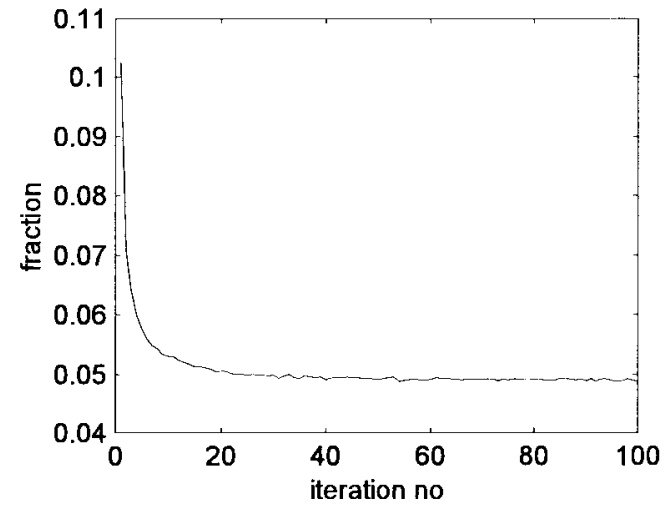

(a)

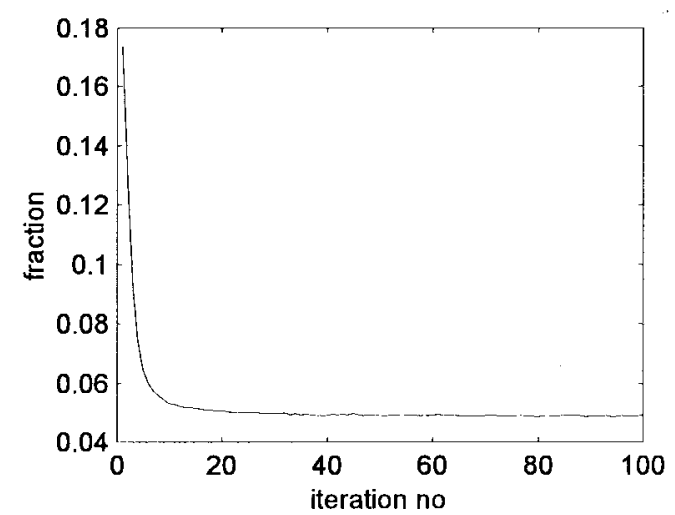

(b)

Fig. 19. Fraction of changed pixels versus iteration number for the iterative error diffusion, starting with (a) the error diffusion error and (b) random error.

sharpening associated with the error diffusion where edges are amplified along one direction only. Also the periodic worms in the uniform regions of Fig. 12 are replaced by more random patterns in Fig. 13. The drawbacks of the error diffusion, edge blurring, and worm artifact, are eliminated using a properly shaped zero phase error filter.

The magnitude response of $1-H\left(\omega_{x}, \omega_{y}\right)$ for the error filter of (18) is shown in Fig. 14. The halftoning noise magnitude spectrum for the halftone of Fig. 13 is shown in Fig. 15. The halftoning noise shape is identical to the magnitude response, as predicted by (8). Fig. 16 compares the radially averaged absolute phase error for the halftone of Fig. 13, with that for the error diffusion halftone of Fig. 12. The plot also includes the mean absolute phase error for the halftone of Fig. 18 described later. Note the consistently low level of mean absolute phase error obtained by the zero phase error filter.

Another example shown in Fig. 17 is the halftone obtained after ten iterations using the same filter, but with starting values of uniform white noise. In a cursory glance Fig. 17 looks similar to Fig. 13, on closer observation one finds that the remaining worms of Fig. 13 have vanished in Fig. 17. The reason behind this is the use of signal-independent noise, which tends to break the worms. Otherwise, the final results are in general independent of the starting error value.

Since any iterative halftoning algorithm may be computationally unattractive for implementation (such as variable number of iterations), one may wish to use the proposed algorithm for a fixed number of passes. Fig. 18 shows the halftone obtained after a single iteration with the identical error filter and starting error values as in Fig. 13. Though not as good as the halftone in Fig. 13, it is in our opinion better than the conventional error diffusion halftone of Fig. 12. This example shows that, even a single pass of the iterative error diffusion approach produces results that we feel are better than the error diffusion approach. Note, however, that Fig. 18 actually requires two passes, the first one being conventional error diffusion to generate the starting error values. It turns out that an overall single pass, such as starting with random noise, does not produce an acceptable result.

Fig. 19 shows the plots of the fraction of changed halftone pixels between two consecutive iterations (used as the convergence criterion), versus the iteration number, for different starting quantization error values. The plots are obtained after averaging the results of five $512 \times 512$ images. The fraction decays relatively slowly for the second case (random starting error), but settles at a smaller value than the first case. Since the starting point in the first case (starting with the error diffusion error) is a good halftone in itself, the convergence is faster. On the other hand, the starting point in the second case is signal independent noise; therefore, it takes more iterations to converge. The settling of the fraction at a smaller value is in accordance with the observed better performance in the second case.

It is seen that the iterative error diffusion algorithm, using a zero phase error filter, produces substantially better halftones (in our opinion) that reproduces even the thin edges of the input. We therefore conclude that the phase response of the error filter is important in order to obtain perceptually good halftones.

\section{ACKNOWLEDGMENT}

The authors wish to acknowledge the suggestions and pointers to prior work received from the reviewers, which have improved the paper considerably.

\section{REFERENCES}

[1] R. W. Floyd and L. Steinberg, "An adaptive algorithm for spatial greyscale," in Proc. Soc. Inf. Disp, 1976, vol. 17, pp. 75-77.

[2] J. F. Jarvis, C. N. Judice, and W. H. Ninke, "A survey of techniques for the display of continuous tone pictures on bilevel displays," in Comput. Graph. Image Processing, 1976, vol. 5, pp. 13-40.

[3] J. C. Stoffel and J. F. Moreland, "A survey of electronic techniques for pictorial image reproduction," IEEE Trans. Commun., vol. COMM-29, pp. 1898-1925, Dec. 1981.

[4] R. A. Ulichney, "Dithering with blue noise," Proc. IEEE, vol. 76, pp. 56-79, Jan. 1988.

[5] E. Barnard, "Optimal error diffusion for computer-generated holograms," J. Opt. Soc. Amer. A, vol. 5, pp. 1803-1817, Nov. 1988.

[6] J. Sullivan, R. Miller, and G. Pios, "Image halftoning using a visual model in error diffusion," J. Opt. Soc. Amer. A, vol. 10, pp. 1714-1724, Aug. 1993.

[7] P. Stucki, "MECCA-A multiple-error correcting computation algorithm for bilevel image hardcopy reproduction,” IBM Res. Rep. RZ1060, 1981.

[8] Z. Fan, "Dot-to-dot error diffusion," J. Electron. Imag., vol. 2, pp. 62-66, Jan. 1993.

[9] __ "Error diffusion with a more symmetric error distribution," Proc. SPIE, vol. 2179, pp. 150-158, May 1994. 
[10] K. T. Knox and R. Eschbach, "Threshold modulation in error diffusion," J. Electron. Imag., vol. 2, pp. 185-192, July 1993.

[11] P. W. Wong, "Adaptive error diffusion and its application in multiresolution rendering," IEEE Trans. Image Processing, vol. 5, pp. 1184-1196, July 1996.

[12] R. Mrusek, M. Broja, and O. Bryngdahl, "Halftoning by carrier and spectrum control," Opt. Commun., vol. 75, pp. 375-380, 1990.

[13] K. T. Knox, "Error image in error diffusion," Proc. SPIE, vol. 1657, pp. 268-279, May 1992.

[14] S. Weissbach and F. Wyrowski, "Error diffusion procedure: Theory and applications in optical signal processing," Appl. Opt., vol. 31, pp. 2518-2534, May 1992.

[15] N. S. Jayant and P. Noll, Digital Coding of Waveforms, Principles and Applications to Speech and Video. Englewood Cliffs, NJ: PrenticeHall, 1984.

[16] S. Kollias and D. Anastassiou, "A unified neural network approach to digital image halftoning," IEEE. Trans. Signal Processing, vol. 39, pp. 980-984, Apr. 1991.

[17] R. Eschbach and K. T. Knox, "Error diffusion algorithm with edge enhancement," J. Opt. Soc. Amer. A, vol. 8, pp. 1844-1850, Dec. 1991

[18] R. Eschbach, "Comparison of error diffusion methods for computergenerated holograms," Appl. Opt., vol. 30, pp. 3702-3710, Sept. 1991.

[19] F. Fetthauer and O. Bryngdahl, "Quantization noise and the error diffusion algorithm," J. Electron. Imag., vol. 3, pp. 37-44, Jan. 1994.

[20] - "On the error diffusion algorithm: Object dependence of the quantization noise," Opt. Comm., vol. 120, pp. 223-229, Nov. 1995.

[21] I. Katsavounidis and C.-C. Jay Kuo, "A multiscale error diffusion technique for digital halftoning," IEEE Trans. Image Processing, vol. 6, pp. 483-490, Mar. 1997.

[22] T. Scheermesser, M. Broja, and O. Bryngdahl, "Adaptation of spectral constraints to electronically halftoned pictures," J. Opt. Soc. Amer. A, vol. 10, pp. 412-417, Mar. 1993.

[23] M. Broja, F. Wyrowski, and O. Bryngdahl, "Significance of spectral phase in synthesis of binarized images," Opt. Commun., vol. 95, pp. 205-209, 1993.

[24] A. Kumar, "Some improvements in error diffusion halftoning of images," Master's Project Rep., Dept. Elect. Commun. Eng., Indian Inst. Sci., Jan. 1996

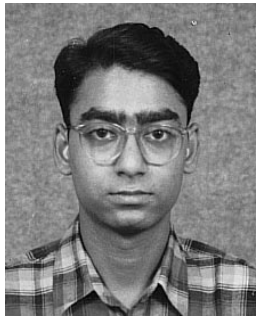

Akhil Kumar received the B.E. degree in electronics from Madan Mohan Malaviya Engineering College, Gorakhpur, India, in 1994, the M.E. degree from the Indian Institute of Science (IISc), Bangalore, in 1996, and completed this work as part of the the M.E. degree in electrical communication engineering at the Visual Communication Laboratory, IISc, Bangalore.

He is currently with IBM Global Services India Limited, Delhi, India. His research interests are in data encryption, image processing, and information technology.

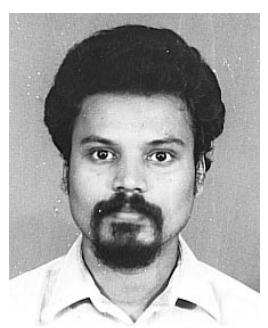

Anamitra Makur received the B.Tech. degree from the Indian Institute of Technology, Kharagpur, in 1985, and the M.S. and Ph.D. degrees in electrical engineering from the California Institute of Technology, Pasadena, in 1986 and 1990.

$\mathrm{He}$ is currently an Associate Professor of electrical communication engineering, Indian Institute of Science, Bangalore. He had a visiting assignment at the University of California, Santa Barbara, during 1997 and 1998. His research interests in signal compression include vector quantization, subband coding and filterbank design, motion field coding, other image/video compression schemes and standards, and multimedia applications. His interests in image/video processing includes halftoning, image restoration, and 2-D filter design.

Dr. Makur is the recipient of the 1998 Young Engineer Award from the Indian National Academy of Engineering. 